CERN-TH/2000-351

hep-th/0011288

\title{
Supergravity and D-branes Wrapping Supersymmetric 3-Cycles
}

\author{
Harald Nieder and Yaron $\mathrm{Oz}$ \\ Theory Division, CERN \\ CH-1211, Geneva, 23, Switzerland
}

\begin{abstract}
We construct dual supergravity descriptions of D3-branes wrapping associative 3 -cycles $L$. We analyse the conditions for having five-dimensional background solutions of the form $A d S_{2} \times L$ and show that they require $L$ to be of constant negative curvature type. This provides $A d S_{2}$ background solutions when $L$ is the hyperbolic space $H^{3}$ or its quotients by subgroups of its isometry group. We construct a regular numerical solution interpolating between $A d S_{5}$ in the UV and $A d S_{2} \times H^{3}$ in the IR. The IR fixed point exists at the "intersection" of the Coulomb and Higgs branches. We analyse the singular supergravity solutions which correspond to moving into the Higgs and the Coulomb branches. For negative constant curvature spaces the singularity is of a "good" type in the Higgs branch and of a "bad" type in the Coulomb branch. For positive constant curvature spaces such as $S^{3}$ the singularity is of a "bad" type in both the Higgs and the Coulomb branches. We discuss the meaning of these results.
\end{abstract}

November 2000 


\section{Introduction}

Supersymmetric 3-cycles are defined such that D-branes wrapping them are supersymmetric configurations $[1,2,3,4]$. We will discuss two types of supersymmetric 3-cycles: associative 3-cycles in $G_{2}$ holonomy seven-dimensional manifolds and special Lagrangian 3-cycles in Calabi-Yau 3-folds ${ }^{1}$. The low-energy field theory on the worldvolume of Dpbranes wrapping associative (special Lagrangian) 3 -cycles is a $(p-2)$-dimensional gauge theory with two (four) supercharges. The Dp-brane worldvolume theory is a twisted field theory [2]. The holonomy group of a 3-cycle $L$ is $\subseteq S O(3)$ and its normal bundle is non-trivial. The scalars parametrizing the embedding of a Dp-brane wrapping $L$ in a the ambient space $M$ are sections of the normal bundle of $L$ in $M$ and are not ordinary scalars. The twisting means choosing the holonomy group of the tangent bundle to be equal to the structure (sub)group of the normal bundle. This gives covariantly constant supercharges which are "twisted" and become scalars rather than spinors.

We will consider Type IIB string theory compactified on $M$ and $N$ D3-branes wrapping the 3 -cycle $L$ in the limit $l_{s} \rightarrow 0$ where we keep the volumes of the cycle $L$ and the manifold $M$ fixed [6]. While at high energies the worldvolume theory is four-dimensional, at low energies compared to the inverse size of $L$ the theory is $(0+1)$-dimensional. We will be interested in the infrared (large time) behaviour of the quantum mechanics.

Our aim is to construct dual supergravity descriptions of the worldvolume theories of the D3-branes wrapped around supersymmetric 3-cycles. Supergravity descriptions of branes wrapping supersymmetric cycles have been constructed in various cases such as for Dp-branes and M5-branes wrapping Riemann surfaces in Calabi-Yau spaces [7, 8, 9, 6, $10,11]$ and M5-branes wrapping associative cycles in $G_{2}$-holonomy manifolds [12]. Here the twist induces the boundary conditions on the gauge field coming from the metric upon compactification [6]. We will use a five-dimensional truncation of the full type IIB supergravity. Since to implement the twist we need an $S O(3)$ gauge potential, one suitable supergravity theory in five dimensions is the $\mathcal{N}=4 S U(2) \times U(1)$ gauged supergravity described in [13] which was shown to be a consistent truncation of type IIB supergravity in [14]. We will see that this gauged supergravity can realize the twist required for D3-branes wrapping an associative 3-cycle.

To analyse what types of 3-cycles we should consider, we note the eight geometric types in the classification of closed 3-manifolds introduced by Thurston [15]. The most relevant for us are the first three types in the classification, based on the three constant curvature spaces, the 3 -sphere $S^{3}$ which has a positive scalar curvature $R>0$ and isometry group $G=S O(4)$, the Euclidean space $E^{3}$ with $R=0$ and isometry group $G=\boldsymbol{R}^{3} \times S O(3)$ and the hyperbolic space $H^{3}$ with $R<0$ and isometry group $G=P S L(2, C)$. The constant curvature spaces $H^{3}, S^{3}$ will be analysed in detail in the following sections. The Euclidean space $E^{3}$, being flat, does not lead to a new background, i.e. the topological twist is trivial.

We will analyse the requirements that an associative 3-cycle $L$ of $S O(3)$ holonomy

\footnotetext{
${ }^{1}$ For a discussion and review of special Lagrangian 3-cycles and their role in "brane worlds" and curved geometries see [5].
} 
has to meet in order to allow for a solution of the form $A d S_{2} \times L$, which corresponds to an IR fixed point. We will see that the cycle has to be of a constant negative scalar curvature type. Thus, we will find an $A d S_{2} \times d s_{H^{3}}^{2}$ solution, and its quotients by subgroups of the isometry group $P S L(2, C)$ to make the 3-cycle compact. Since the spinors are independent of the coordinates on $H^{3}$ these quotient solutions are also supersymmetric. We will construct a regular numerical solution interpolating between $A d S_{5}$ in the UV and $A d S_{2} \times H^{3}$ in the IR. The IR fixed point exists at the "intersection" of the Coulomb and Higgs branches. We will analyse the singular supergravity solutions which correspond to moving into the Higgs and the Coulomb branches. For negative constant curvature spaces the singularity will be shown to be of a "good" type in the Higgs branch and of a "bad" type in the Coulomb branch. This indicates a decoupling of the Higgs branch from the Coulomb branch. For positive constant curvature spaces such as $S^{3}$ the singularity is of a "bad" type in both the Higgs and the Coulomb branches, which is consistent with the absence of a Higgs branch on the field theory side in this case.

The paper is organized as follows. In section 2 we will discuss in some detail the twisted theories on the worldvolume of D3-branes wrapping associative and special Lagrangian 3cycles. We will review the barely $G_{2}$ manifolds and discuss their supersymmetric 3-cycles. In section 3 we will discuss the supergravity equations which have to be solved in order to provide supergravity dual descriptions of the twisted D3-branes theories. In section 4 we will begin by reviewing the possible types of 3-cycles that should be analysed. We will then construct solutions to the supergravity equations. We will show that supersymmetric $A d S_{2} \times L$ backgrounds are solutions provided the associative 3-cycle is of a constant negative scalar curvature type. However, we will show that wrapping associative 3-cycles of the type $\Sigma \times S^{1}$ is not realized by the $\mathcal{N}=4$ gauged supergravity. We will solve numerically the supergravity equations and construct a regular solution interpolating between $A d S_{5}$ in the UV and $A d S_{2} \times H^{3}$ in the IR. We will next analyse the singular supergravity solutions which correspond to moving into the Higgs and the Coulomb branches and the type of the singularities. In appendix A we will show how the case of D3-branes wrapping a Riemann surface arises from our construction and discuss the nature of the singularities along the Coulomb and Higgs branches. In appendix B we will provide some details of the computations for $H^{3}$ and $S^{3}$ special associative 3-cycles.

\section{D3-branes wrapping supersymmetric 3-cycles}

In this section we will discuss two types of supersymmetric 3-cycles, the associative 3cycle in $G_{2}$ holonomy seven-dimensional manifolds and the special Lagrangian 3-cycle in Calabi-Yau 3-fold. We will review the example of barely $G_{2}$ manifolds constructed as the quotients $\left(C Y_{3} \times S^{1}\right) / Z_{2}$, where one can see a relation between the supersymmetric 3cycles of the seven dimensional manifold and the supersymmetric cycles of the Calabi-Yau 3 -fold. We will analyse the twists associated with D3-branes wrapping the 3-cycles and discuss the corresponding twisted worldvolume theories. We note that in the following we will freely use $S U(2) \simeq S O(3)$. 


\subsection{Associative 3-cycles}

An associative 3 -cycle $L$ is a submanifold of a seven dimensional manifold with $G_{2}$ holonomy. It is a calibrated submanifold with respect to the associative calibration 3-form $\Phi_{(3)}$ [16]. That means that the associative 3-cycles are volume minimizing in their homology class such that

$$
\left.\Phi_{(3)}\right|_{L}=V o l_{L} .
$$

They are supersymmetric cycles in the sense that D-branes wrapping them are supersymmetric configurations [4]. Consider Type IIB string theory compactified on a seven dimensional manifold with $G_{2}$ holonomy $M$, and a D3-brane wrapping $L$. The brane configuration is a BPS state of Type IIB string theory compactified on $M$. It is a particle in the transverse $R^{2,1}$ directions. The D3-brane worldvolume theory is a twisted field theory. The holonomy group of the 3-cycle $L$ is $\subseteq S O(3)$ and its normal bundle $\mathcal{N}$ in $M$ is $S \otimes V$ [17], where $S$ is a spin bundle and $V$ a vector bundle. The four scalars parametrizing the embedding of the D3-brane in $M$ are sections of the normal bundle of $L$ in $M$ and are therefore not ordinary scalars. Let us analyse this structure in more detail.

The symmetry group of a D3-brane in flat space is $S O(3,1) \times S O(6)$, where $S O(3,1)$ is the Lorentz symmetry and $S O(6)$ is the R-symmetry group of the worldvolume theory. Alternatively, they can be viewed as the symmetry groups of the tangent and normal bundles respectively. The worldvolume of the brane is $R \times L$ and the worldvolume theory is a twisted theory. Due to the wrapping the structure group of the normal bundle is broken to $U(1) \times S U(2)_{1} \times S U(2)_{2}$ and

$$
S O(3,1) \times S O(6) \rightarrow S O(3)_{L} \times U(1) \times S U(2)_{1} \times S U(2)_{2} .
$$

Here $S O(3)_{L}$ is the structure group of the tangent bundle of $L$.

The six scalars parametrizing the embedding of the D3-brane in flat space transform as $(\mathbf{1}, \mathbf{6})$ under $S O(3,1) \times S O(6)$. The $\mathbf{6}$ of the $S O(6)$ transforms under $U(1) \times S U(2)_{1} \times$ $S U(2)_{2}$ as $(\mathbf{1}, \mathbf{1})_{ \pm \mathbf{2}} \oplus(\mathbf{2}, \mathbf{2})_{\mathbf{0}}$, where the subscript indicates the $U(1)$ charge. The twisting means choosing the holonomy group of the tangent bundle $S O(3)_{L}$ to be equal to the $S U(2)_{1}$ part of the structure group of the normal bundle. After the twisting the six scalars transform as $(\mathbf{1}, \mathbf{1})_{ \pm \mathbf{2}} \oplus(\mathbf{2}, \mathbf{2})_{\mathbf{0}}$ under $U(1) \times S U(2)_{L} \times S U(2)_{2}$. The $(\mathbf{2}, \mathbf{2})_{\mathbf{0}}$ parametrize the embedding of the D3-brane inside the $G_{2}$ manifold, and are sections of the normal bundle described above. The $(\mathbf{1}, \mathbf{1})_{ \pm \mathbf{2}}$ are the two scalars that parametrize the embedding of the D3-brane in the two non-compact flat directions normal to the brane.

The sixteen supercharges of the D3-brane in flat space transform as $(\mathbf{2}, \mathbf{1}, \mathbf{4}) \oplus(\mathbf{1}, \mathbf{2}, \overline{\mathbf{4}})$ under $S O(3,1) \times S O(6)$. The 4 of $S O(6)$ transforms under $U(1) \times S U(2)_{1} \times S U(2)_{2}$ as $(\mathbf{2}, \mathbf{1})_{\mathbf{1}} \oplus(\mathbf{1}, \mathbf{2})_{-\mathbf{1}}$. After the twist we remain with two covariantly constant supercharges which transform under $U(1) \times S U(2)_{L} \times S U(2)_{2}$ as $(\mathbf{1}, \mathbf{1})_{ \pm \mathbf{1}}$. The supercharges are "twisted" and become scalars rather than spinors.

We will consider $N$ D3-branes wrapping the 3-cycle $L$ in the limit $l_{s} \rightarrow 0$ where we keep the volumes of the cycle $L$ and the $G_{2}$ manifold $M$ fixed [6]. This is the large volume limit in string units. Due to the non-trivial embedding of the cycle in $M$ the worldvolume 
theory in this limit is a twisted field theory, as described above. However, note that in this limit the theory is not sensitive to the global structure of $M$.

While at high energies the worldvolume theory is four-dimensional, at low energies compared to the inverse size of $L$ the theory is $(0+1)$-dimensional $U(N)$ gauge theory. The $(0+1)$-dimensional gauge coupling $g$ is given by $g^{2}=\frac{g_{s}}{V o l(L)}$. It has a mass dimension $\frac{3}{2}$. We will be interested in the infrared (large time) behaviour of the quantum mechanics. At low-energies the theory is described by a supersymmetric $\sigma$-model quantum mechanics with two supercharges. The target space has branches which we denote by Higgs and Coulomb, and is not protected from quantum corrections.

The Higgs branch corresponds to the moduli of the associative 3-cycle embedded in the $G_{2}$ manifold. The Coulomb branch corresponds to the moduli of the D-branes in the two transverse non-compact flat directions. At low energies we may expect a decoupling of the Higgs and Coulomb branches, such that the IR physics is that of a supersymmetric $\sigma$-model quantum mechanics on the Higgs branch. This is argued for theories with eight supercharges in $[18,19]$ and for theories with four supercharges in [20]. We will see that the dual supergravity description indicates a decoupling of the Higgs and Coulomb branches in our case, which has only two supercharges.

\subsection{Special Lagrangian 3-cycles}

Here we consider Type IIB string theory compactified on a Calabi-Yau 3-fold $M$. Denote by $\kappa$ the Kaehler form of $M$ and by $\Omega$ the holomorphic $(3,0)$ form. A 3-cycle $L \in M$ is called special Lagrangian when

(i) $\left.\kappa\right|_{L}=0$

(ii) $\left.\operatorname{Im}(\Omega)\right|_{L}=0$.

Consider a D3-brane wrapping $L$. Again, the D3-brane worldvolume theory is a twisted field theory. The holonomy group of the 3-cycle $L$ is $\subseteq S O(3)$ and its normal bundle $\mathcal{N}$ in $M$ can be identified with the cotangent bundle $T^{*} L$. The three scalars parametrizing the embedding of the D3-brane in $M$ are sections of the normal bundle of $L$ in $M$ and are therefore 1-forms rather than ordinary scalars. Due to the wrapping the symmetry group is broken as

$$
S O(3,1) \times S O(6) \rightarrow S O(3)_{L} \times S O(3)_{\mathcal{N}} \times S O(3)_{T} .
$$

Here $S O(3)_{L}$ is the symmetry group of $L, S O(3)_{\mathcal{N}}$ is the structure group of the normal bundle of $L$ in $M, S O(3)_{T}$ is the symmetry group of the three non-compact flat directions transverse to the brane.

The sixteen supercharges of the D3-brane in flat space transform as $(\mathbf{2}, \mathbf{1}, \mathbf{4}) \oplus(\mathbf{1}, \mathbf{2}, \overline{\mathbf{4}})$ under $S O(3,1) \times S O(6)$. Since the $\mathbf{4}$ of $S O(6)$ transforms under a subgroup $S O(3)_{\mathcal{N}} \times$ $S O(3)_{T}$ as $(\mathbf{2}, \mathbf{2})$, the supercharges transform under $S O(3)_{L} \times S O(3)_{\mathcal{N}} \times S O(3)_{T}$ as $(\mathbf{2}, \mathbf{2}, \mathbf{2}) \oplus(\mathbf{2}, \mathbf{2}, \mathbf{2})$. The twisting means choosing the holonomy group of the tangent bundle $S O(3)_{L}$ to be equal to the structure group of the normal bundle $S O(3)_{\mathcal{N}}$. This gives four covariantly constant supercharges which transform as $2(\mathbf{1}, \mathbf{2})$ under $S O(3)_{L} \times S O(3)_{T}$. The supercharges are "twisted" and become scalars rather than spinors. 
The six scalars parametrizing the embedding of the D3-brane in flat space transform as $(\mathbf{1}, \mathbf{6})$ under $S O(3,1) \times S O(6)$. Since the $\mathbf{6}$ of $S O(6)$ transforms under a subgroup $S O(3)_{\mathcal{N}} \times S O(3)_{T}$ as $(\mathbf{1}, \mathbf{3}) \oplus(\mathbf{3}, \mathbf{1})$, the six scalars transform under $S O(3)_{L} \times S O(3)_{\mathcal{N}} \times$ $S O(3)_{T}$ as $(\mathbf{1}, \mathbf{1}, \mathbf{3}) \oplus(\mathbf{1}, \mathbf{3}, \mathbf{1})$. After the twist they transform as $(\mathbf{1}, \mathbf{3}) \oplus(\mathbf{3}, \mathbf{1})$ under $S O(3)_{L} \times S O(3)_{T}$. The $(\mathbf{1}, \mathbf{3})$ are the three scalars that parametrize the embedding of the D3-brane in the three non-compact flat directions normal to the brane. The $(\mathbf{3}, \mathbf{1})$ correspond to the 1-form that parametrizes the embedding of the D3-brane in the directions normal to the brane in $M$.

We would like to consider $N$ D3-branes wrapping the 3 -cycle $L$ in the above limit. At low-energies the theory is described by a supersymmetric $\sigma$-model quantum mechanics with four supercharges. The Higgs branch corresponds to the moduli of the special Lagrangian cycle embedded in the Calabi-Yau 3-fold. The Coulomb branch corresponds to the moduli of the D-branes in the three transverse non-compact flat directions. Again, we may expect a decoupling of the Higgs and Coulomb branches in the IR.

\subsection{Barely $G_{2}$ manifolds}

Let us illustrate the associative and special Lagrangian 3-cycles by discussing the barely $G_{2}$ manifolds [21]. These are constructed as

$$
G=\left(C Y_{3} \times S^{1}\right) / Z_{2}
$$

where $C Y_{3}$ is the Calabi-Yau 3-fold and $Z_{2}$ acts as $(\sigma,-1) . \quad \sigma$ is an anti-holomorphic involution on the Calabi-Yau 3-fold. Its action on the Kaehler form $\kappa$ of $C Y_{3}$, and on the holomorphic $(3,0)$ form $\Omega$ is

$$
\sigma^{*}(\kappa)=-\kappa, \quad \sigma^{*}(\Omega)=\bar{\Omega} .
$$

There is only one covariantly constant spinor on $G$, which corresponds to the linear combination of the two covariantly constant spinors with opposite chirality on $C Y_{3}$.

The associative calibration 3 -form reads [21]

$$
\Phi_{(3)}=J \wedge e^{1}+R e[\Omega],
$$

where $e^{1}$ is a one-form cotangent to the circle $S^{1}$.

There are two types of supersymmetric associative 3 -cycles $\mathcal{C}_{3}$. The first one is constructed as a product of $C \times S^{1}$ where $C$ is a holomorphic cycle in $C Y_{3}$, modded by the action of $Z_{2}$

Note that $\sigma$ maps $C$ to $-C$.

$$
\mathcal{C}_{3}=\left(C \times S^{1}\right) / Z_{2}
$$

The second type is constructed by the quotient of a special Lagrangian cycle $L$ in $C Y_{3}$

$$
\mathcal{C}_{3}=L / Z_{2} \text {. }
$$

For this type of associative 3-cycles the calibration form is just $R e[\Omega]$ as for a special Lagrangian 3-cycle. Note, however, that the ambient space in which the 3-cycle is embedded is different, and the number of supersymmetries preserved by the associative 3-cycle is half that of the special Lagrangian one. 


\section{The supergravity equations}

We aim at a dual supergravity description of the worldvolume theory of $N$ D3-branes wrapped around a supersymmetric 3-cycle $L$. As discussed above, the worldvolume theory is twisted, and the twist induces the boundary conditions on the gauge field coming from the metric upon compactification. It also implies that a scalar operator in the $\mathbf{2 0}$ representation of $S O(6)$ is turned on, as in [6].

We will use a five-dimensional truncation of the full type IIB supergravity. To find a suitable truncation we observe that the holonomy of the $L$ is $S O(3)$ so for the twist we will need an $S O(3)$ gauge potential. One suitable supergravity theory in five dimensions is the $\mathcal{N}=4 S U(2) \times U(1)$ gauged supergravity described in [13] which was shown to be a consistent truncation of type IIB supergravity in [14]. The embedding of this fivedimensional supergravity in type IIB supergravity is done via the breaking $S O(6) \rightarrow$ $U(1) \times S U(2)_{1} \times S U(2)_{2}$, where $S U(2) \times U(1)$ of the $\mathcal{N}=4$ is the $S U(2)_{1} \times U(1)$. From the discussion of the previous section we know that this can realize the twist required for D3-branes wrapping an associative 3-cycle.

We are looking for a supersymmetric solution of the theory of [13]. To this end we have to check the supersymmetric variations of the fermionic fields. These are given by

$\delta \psi_{\mu a}=D_{\mu} \varepsilon_{a}+i \gamma_{\mu} T\left(\Gamma_{45}\right)_{a b} \varepsilon^{b}+i \frac{1}{6} \sqrt{\frac{1}{2}}\left(\gamma_{\mu}^{\nu \rho}-4 \delta_{\mu}^{\nu} \gamma^{\rho}\right)\left(H_{\nu \rho a b}+\sqrt{\frac{1}{2}} h_{\nu \rho a b}\right) \varepsilon^{b}$,

for the four gravitini and

$$
\delta \chi_{a}=-i \sqrt{\frac{1}{2}} \gamma^{\mu}\left(\partial_{\mu} \Phi\right) \varepsilon_{a}+A\left(\Gamma_{45}\right)_{a b} \varepsilon^{b}+\frac{1}{2} \sqrt{\frac{1}{6}} \gamma^{\mu \nu}\left(H_{\mu \nu a b}-\sqrt{2} h_{\mu \nu a b}\right) \varepsilon^{b}
$$

for the remaining four spin- $\frac{1}{2}$ fields. The notation agrees with [13] except that we use a space-time metric with signature $(-,+,+,+,+)$. The definitions are

$$
\begin{aligned}
A & \equiv \frac{1}{2} \sqrt{\frac{1}{6}} g_{2} \xi^{-1}-\frac{1}{2} \sqrt{\frac{1}{3}} g_{1} \xi^{2}, \\
T & \equiv \frac{1}{6} \sqrt{\frac{1}{2}} g_{2} \xi^{-1}+\frac{1}{12} g_{1} \xi^{2}, \\
h_{\mu \nu a b} & \equiv-\xi^{-2} \Omega_{a b} f_{\mu \nu}, \\
H_{\mu \nu a b} & \equiv \xi\left(F_{\mu \nu}^{I}\left(\Gamma_{I}\right)_{a b}+B_{\mu \nu}^{\alpha}\left(\Gamma_{\alpha}\right)_{a b}\right), \\
\xi & \equiv \exp \sqrt{\frac{2}{3}} \Phi .
\end{aligned}
$$

The $\gamma_{\nu}, \nu=0, \ldots, 4$ are the five dimensional space-time gamma matrices, whereas the $\Gamma_{I, \alpha}$ are the $\operatorname{Spin}(5)$ gamma matrices with the index ranges $I=1,2,3$ and $\alpha=4,5$. The $a, b$ are $\operatorname{Spin}(5)$ spinor indices and $\Omega_{a b}$ is the antisymmetric matrix used to lower these indices. $F_{\mu \nu}^{I}$ and $f_{\mu \nu}$ are the $S U(2)$ and $U(1)$ gauge fields respectively, $g_{2}$ and $g_{1}$ are the 
corresponding coupling constants, $\Phi$ is a scalar field and $B_{\mu \nu}^{\alpha}$ are antisymmetric tensor fields transforming as a $U(1)$ doublet.

The $S U(2) \times U(1)$ is embedded in $\operatorname{Spin}(5)$ by taking $\Gamma_{I 45}$ and $\Gamma_{45}$ as the respective generators. We therefore have the covariant derivative on the spinors $\varepsilon_{a}$

$$
D_{\mu} \varepsilon_{a}=\nabla_{\mu} \varepsilon_{a}+\frac{1}{2} g_{1} a_{\mu}\left(\Gamma_{45}\right)_{a}^{b} \varepsilon_{b}+\frac{1}{2} g_{2} A_{\mu}^{I}\left(\Gamma_{I 45}\right)_{a}^{b} \varepsilon_{b}
$$

with $\nabla_{\mu}$ denoting the usual space-time covariant derivative and $a_{\mu}, A_{\mu}^{I}$ are the $U(1), S U(2)$ gauge potentials. Furthermore we will use the freedom to rescale the coupling constants as in [13] and define $\bar{g} \equiv \sqrt{2} g_{1}, g_{2} \equiv \bar{g}$. Note that we cannot set the $U(1)$ coupling $g_{1}$ simply to zero since the kinetic term of the $B_{\mu \nu}^{\alpha}$ field goes like $\frac{1}{g_{1}}[13]^{2}$.

For later reference it is useful to recall the five-dimensional field equations for zero fermionic fields which follow from the Lagrangian given in [13]. In terms of our conventions and with $a_{\mu}, B_{\mu \nu}^{\alpha}$ set to zero we obtain for the metric

$$
\mathcal{R}_{\mu \nu}-3 \partial_{\mu} \varphi \partial_{\nu} \varphi-2 e^{2 \varphi} F_{\mu \alpha}^{I} F_{\nu I}^{\alpha}+\frac{1}{3} e^{2 \varphi} F^{2} g_{\mu \nu}+\frac{4}{3} P(\varphi) g_{\mu \nu}=0,
$$

and for the scalar

$$
\nabla^{2} \varphi-\frac{1}{3} e^{2 \varphi} F^{2}+\frac{2}{3} \frac{\partial P(\varphi)}{\partial \varphi}=0
$$

where for notational simplicity we introduced $\varphi \equiv \sqrt{\frac{2}{3}} \Phi$ and used $F^{2}$ as short for $F_{\mu \nu}^{I} F_{I}^{\mu \nu}$. The scalar potential $P(\varphi)$ reads

$$
P(\varphi)=\frac{1}{8} \bar{g}^{2}\left(e^{-2 \varphi}+2 e^{\varphi}\right)
$$

For a supersymmetric solution the variations $(9),(10)$ of the fermionic fields must be zero. As the five dimensional metric we take the ansatz

$$
d s^{2}=e^{2 f}\left(-d t^{2}+d r^{2}\right)+e^{2 g} d s_{L}^{2},
$$

where by $d s_{L}^{2}$ we denote the metric on the 3 -cycle $L$

$$
d s_{L}^{2}=g_{i j} d x^{i} d x^{j}
$$

We assume the functions $f, g$ to depend only on the radial coordinate $r$. We also take the scalar field $\varphi$ to depend only on $r . \varphi$ is dual to the turned on scalar operator.

Thus, we have to solve the following equations

$$
\begin{aligned}
& \delta \psi_{\mu a}=\partial_{\mu} \varepsilon_{a}+\frac{1}{4} \omega_{\mu}^{i j} \gamma_{i j} \varepsilon_{a}+\frac{1}{2} \bar{g} A_{\mu}^{I}\left(\Gamma_{I 45}\right)_{a}^{b} \varepsilon_{b}- \\
& -i \gamma_{\mu} T\left(\Gamma_{45}\right)_{a}^{b} \varepsilon_{b}-i \frac{1}{6} \sqrt{\frac{1}{2}}\left(\gamma_{\mu}^{\nu \rho}-4 \delta_{\mu}^{\nu} \gamma^{\rho}\right) e^{\varphi} F_{\nu \rho}^{I}\left(\Gamma_{I}\right)_{a}^{b} \varepsilon_{b}=0
\end{aligned}
$$

\footnotetext{
${ }^{2}$ A supergravity action where the $U(1)$ coupling can be taken to zero has been constructed in [22].
} 


$$
\delta \chi_{a}=-i \frac{\sqrt{3}}{2} \gamma^{r}\left(\partial_{r} \varphi\right) \varepsilon_{a}-A\left(\Gamma_{45}\right)_{a}^{b} \varepsilon_{b}-\frac{1}{2} \sqrt{\frac{1}{6}} \gamma^{\mu \nu} e^{\varphi} F_{\mu \nu}^{I}\left(\Gamma_{I}\right)_{a}^{b} \varepsilon_{b}=0,
$$

with the definitions

$$
\begin{aligned}
A & \equiv \frac{1}{2} \sqrt{\frac{1}{6}} \bar{g}\left(e^{-\varphi}-e^{2 \varphi}\right), \\
T & \equiv \frac{1}{12 \sqrt{2}} \bar{g}\left(2 e^{-\varphi}+e^{2 \varphi}\right) .
\end{aligned}
$$

We note that for a time independent spinor $\varepsilon_{a}$ we can combine the $t$-component of equations (22) and (23) to get the relation

$$
f^{\prime}-\varphi^{\prime}= \pm \frac{\bar{g}}{2 \sqrt{2}} e^{f} e^{2 \varphi},
$$

for $\left(\Gamma_{45}\right)_{a}^{b} \varepsilon_{b}=\mp i \varepsilon_{a}$. This relation is independent of the metric of $L$.

At the boundary of $A d S_{5}$ (small $r$ ) we impose the metric

$$
d s^{2} \sim \frac{-d t^{2}+d r^{2}+d s_{L}^{2}}{r^{2}} .
$$

This implies at small $r$ the boundary conditions $f(r), g(r) \sim-\log (r)$. Note that we work in the units where the $A d S_{5}$ radius is one. In order to restore the units and the $N$ dependence we have to multiply the metric by $R_{A d S}^{2}=\sqrt{4 \pi g_{s} N} \alpha^{\prime}$.

We impose the condition

$$
\gamma_{1} \varepsilon_{a}=\varepsilon_{a},
$$

where the subscripts are flat indices associated to the space-time indices according to (83). This condition is met by (super)covariantly constant spinors on $A d S$ which depend only on $r[23,24]$.

To realize the twist we have to satisfy

$$
\frac{1}{4} \omega_{\hat{\mu}}^{\hat{i} \hat{j}} \gamma_{\hat{i} \hat{j}} \varepsilon_{a}=-\frac{1}{2} \bar{g} A_{\hat{\mu}}^{I}\left(\Gamma_{I 45}\right)_{a}^{b} \varepsilon_{b},
$$

where the hatted indices are ( $\hat{\mu}$ curved, $\hat{i}$ flat) indices along $L$. We do this by appropriately mapping the generators of the holonomy group to the generators of the $S U(2)$ gauge group and letting the $U(1)$ generator $\Gamma_{45}$ act on the spinors as $\left(\Gamma_{45}\right)_{a}^{b} \varepsilon_{b}= \pm i \varepsilon_{a}$. The boundary conditions on the metric imply that the positive sign is the correct choice as can be seen from the gravitino variation (9) in $t$ direction. By equating the spin connection with the gauge connection we thus get

$$
\gamma_{\hat{i} \hat{j}} \varepsilon_{a} \propto i\left(\Gamma_{f(\hat{i}, \hat{j})}\right)_{a}^{b} \varepsilon_{b},
$$

where $f$ establishes the map between the generators of the two groups (see below and appendix). 


\section{Supergravity backgrounds}

In this section we will construct analytical and numerical solutions to the supergravity equations.

\subsection{Three manifolds}

In the following we discuss what types of 3-cycles we should consider. Thurston introduced eight geometric types in the classification of closed 3-manifolds [15], which we will briefly review ${ }^{3}$. The main points of the classification are that there are only eight basic homogeneous geometries, up to an equivalence relation, that can be supported by closed 3-manifolds and that if a closed 3-manifold of a given topology admits one of these geometric types then it is unique.

One considers an orientable, connected, complete and simply connected Riemannian 3 -manifold $X$ which is homogeneous with respect to an orientation preserving group of isometries $G$. The eight geometric types classify $(X, G)$. The equivalence relation $(X, G) \sim\left(X^{\prime}, G^{\prime}\right)$ holds when there is a diffeomorphism of $X$ onto $X^{\prime}$ which takes the action of $G$ onto the action of $G^{\prime}$. Out of these types one constructs spaces (geometric structures) $M \simeq X / \Gamma$ where $\Gamma$ is a subgroup of $G$. Here the action of $\Gamma$ is discontinuous, discrete and free. $M$ is locally homogeneous with respect to the metric on $(X, G)^{4}$, it is isometric to the quotient of $X$ by $\Gamma$.

The first three types in the classification are based on the three constant curvature spaces, the 3 -sphere $S^{3}$ which has a positive scalar curvature $R>0$ and isometry group $G=S O(4)$, the Euclidean space $E^{3}$ with $R=0$ and isometry group $G=\boldsymbol{R}^{3} \times S O(3)$ and the hyperbolic space $H^{3}$ with $R<0$ and isometry group $G=P S L(2, C)$. The constant curvature spaces $S^{3}, H^{3}$ will be analysed in detail in the following sections. The Euclidean space $E^{3}$, being flat, does not lead to a new background, i.e. the topological twist is trivial. This class includes, for instance, the 3-torus $T^{3}$.

The next two types are based on $S^{2} \times E^{1}$ and $H^{2} \times E^{1}$. In this class we have, for instance, $\Sigma_{g} \times S^{1}$ where $\Sigma_{g}$ is a genus $g$ Riemann surface.

Type six is the universal cover of the fiber bundle whose base is $H^{2}$ and whose fiber is spanned by tangent vectors of unit length. The last two types are the Heisenberg group and the solvable Lie group. These types are anisotropic geometries and one can write an explicit metric for them [25]. However, it is not clear whether any of these last three types can be realized as a supersymmetric 3-cycle.

\footnotetext{
${ }^{3}$ For the relation between Thurston classification and the classification of spatially homogeneous metrics in relativistic cosmology see [25].

${ }^{4} M$ is called locally homogeneous if for any two points in $M$ there are neighborhoods of these two points and an isometry that maps them to each other.
} 


\section{2 $A d S_{2} \times L$ solutions}

In this subsection we analyse the requirements an associative 3-cycle with $S O(3)$ holonomy has to meet in order to allow for a solution of the form $A d S_{2} \times L$, which corresponds to an IR fixed point. We will see that for our ansatz $L$ has to be of constant negative curvature type. We consider the ansatz $(20),(21)$. Due to the twist we can establish a relation between the $S U(2)$ gauge field strength and the curvature of $L$

$$
F_{\mu \nu}^{I}\left(\Gamma_{I}\right)_{a}^{b} \varepsilon_{b}=i \frac{1}{2 \bar{g}} R_{\mu \nu}^{i j} \gamma_{i j} \varepsilon_{a}
$$

which follows from (28). We now take the variation of the gravitino (22) which is polarized in the $t$ direction. This may be written as

$$
\left(\frac{1}{2} f^{\prime}+e^{f} \frac{1}{12 \sqrt{2}} \bar{g}\left(2 e^{-\varphi}+e^{2 \varphi}\right)+\frac{1}{6} \sqrt{\frac{1}{2}} e^{f} e^{\varphi} \frac{1}{2 \bar{g}} \gamma^{\nu \rho} R_{\nu \rho}^{i j} \gamma_{i j}\right) \gamma_{0} \varepsilon_{a}=0,
$$

so that

$$
\left(\frac{1}{2} f^{\prime}+e^{f} \frac{1}{12 \sqrt{2}} \bar{g}\left(2 e^{-\varphi}+e^{2 \varphi}\right)\right) \gamma_{0} \varepsilon_{a}=-\frac{1}{6} \sqrt{\frac{1}{2}} e^{f} e^{\varphi} \frac{1}{2 \bar{g}} \gamma^{\nu \rho} R_{\nu \rho}^{i j} \gamma_{i j} \gamma_{0} \varepsilon_{a}
$$

The lhs of (32) depends only on $r$ so the rhs must be independent of the coordinates on $L$. The rhs depends on the coordinates on $L$ through the term $\gamma^{\nu \rho} R_{\nu \rho}^{i j} \gamma_{i j}$ so that we have the condition

$$
\gamma^{\nu \rho} R_{\nu \rho}^{i j} \gamma_{i j}=\text { const }
$$

up to an $r$ dependent term coming from the vielbein needed to change the curved indices in $\gamma^{\nu \rho}$ to flat ones. In three dimensions the Weyl tensor vanishes identically so that the Riemann tensor can be expressed in terms of Ricci tensor and scalar curvature

$$
R_{\kappa \lambda \mu \nu}=R_{\kappa \mu} g_{\lambda \nu}-R_{\lambda \mu} g_{\kappa \nu}+R_{\lambda \nu} g_{\kappa \mu}-R_{\kappa \nu} g_{\lambda \mu}-\frac{R}{2}\left(g_{\kappa \mu} g_{\lambda \nu}-g_{\kappa \nu} g_{\lambda \mu}\right) .
$$

Using this in (33) we see that the scalar curvature has to be constant. For cycles with $S O(3)$ holonomy this means that they are maximally symmetric cycles which satisfy

$$
R_{\kappa \lambda \mu \nu}=\frac{R}{6}\left(g_{\kappa \mu} g_{\lambda \nu}-g_{\kappa \nu} g_{\lambda \mu}\right)
$$

We are now in the position to investigate (supersymmetric) solutions of the equations (22) and (23) which are of the form $A d S_{2} \times L$. We require

$$
e^{2 f}=\frac{A^{2}}{r^{2}}
$$

according to the ansatz (20). We rewrite (31) for the maximally symmetric cycle by using (36). This leads to

$$
\frac{1}{6 \bar{g}} A \sqrt{\frac{1}{2}} e^{-2 g} R=-\frac{1}{2} e^{-\varphi}+A \frac{\bar{g}}{12 \sqrt{2}}\left(2 e^{-2 \varphi}+e^{\varphi}\right) .
$$


In order to satisfy equation (25) with the boundary condition (36) $\varphi$ has to approach a constant value in the far IR $(r \rightarrow \infty)$. Equation $(37)$ then also requires $e^{2 g}$ to be asymptotically constant. So supersymmetric solutions in the IR with an $A d S_{2}$ part are indeed of the product form $A d S_{2} \times L$ where $L$ has constant size.

We can plug this additional information into the remaining fermion variations of $(22)$ and (23). This results in

$$
R=-\frac{1}{2} e^{2 g} \bar{g}^{2}\left(2 e^{-2 \varphi}+e^{\varphi}\right)
$$

and

$$
R=e^{2 g} \bar{g}^{2}\left(e^{-2 \varphi}-e^{\varphi}\right) .
$$

Combining these two equations and using (37) we find

$$
e^{3 \varphi}=4, e^{2 g}=-\frac{1}{3 \bar{g}^{2}} 4^{\frac{2}{3}} R, \quad e^{2 f}=\frac{4^{\frac{2}{3}}}{2 \bar{g}^{2} r^{2}},
$$

where $R$ is the scalar curvature of $L$. From (38) and (40) we see that the the scalar curvature of $L$ has to be negative. We conclude that for a supersymmetric solution of the type $A d S_{2} \times L$ the 3 -cycle $L$ has to be of negative constant curvature type. The solution preserves two supercharges.

Thus, there is an $A d S_{2} \times d s_{H^{3}}^{2}$ solution and we can take its quotients by the isometry group $P S L(2, C)$ to make the 3-cycle compact. Since the spinors are independent of the coordinates on $H^{3}$ these quotient solutions are supersymmetric too. If we take the $d s_{H^{3}}^{2}$ line element

$$
d s_{H^{3}}^{2}=d \phi^{2}+\sinh ^{2} \phi d \theta^{2}+\sinh ^{2} \phi \sin ^{2} \theta d \nu^{2},
$$

we get

$$
e^{3 \varphi}=4, e^{2 g}=4^{-\frac{1}{3}}, e^{2 f}=\frac{1}{4^{\frac{4}{3}} r^{2}},
$$

where we have set $\bar{g}=2 \sqrt{2}$.

Using [14] we can lift the five-dimensional background to ten dimensions. The tendimensional metric reads

$$
\begin{aligned}
& d s_{10}^{2}=\sqrt{\Delta}\left(\frac{1}{4^{\frac{4}{3}} r^{2}} d s_{2}^{2}+4^{-\frac{1}{3}} d s_{H^{3}}^{2}\right)+ \\
& +\frac{1}{8} 2^{\frac{1}{3}} \sqrt{\Delta} d \xi^{2}+\frac{1}{8} 2^{-\frac{1}{3}} \Delta^{-\frac{1}{2}} \sin ^{2} \xi d \tau^{2}+ \\
& +\frac{1}{8} 2^{-\frac{1}{3}} \Delta^{-\frac{1}{2}} \cos ^{2} \xi\left\{\left(\sigma_{1}-\cosh \phi d \theta\right)^{2}+\left(\sigma_{2}-\cos \theta d \nu\right)^{2}+\left(\sigma_{3}+\sin \theta \cosh \phi d \nu\right)^{2}\right\},
\end{aligned}
$$

where $(\xi, \tau, \alpha, \beta, \gamma)$ parametrize the compactifying $S^{5}$ and the 1-forms $\sigma_{i}$ are related to the angles $(\alpha, \beta, \gamma)$ by $\sigma_{1}+i \sigma_{2}=e^{-i \gamma}(d \alpha+i \sin \alpha d \beta)$ and $\sigma_{3}=d \gamma+\cos \alpha d \beta$. Furthermore

$$
\Delta=4^{\frac{2}{3}} \sin ^{2} \xi+4^{-\frac{1}{3}} \cos ^{2} \xi
$$


where $d s_{2}^{2}=-d t^{2}+d r^{2}$.

It is illuminating take a closer a look at the field equations (17) and (18). From (17) we get for the Ricci Scalar $\mathcal{R}$ of the five-dimensional background

$$
\mathcal{R}=3(\partial \varphi)^{2}+\frac{1}{3} e^{2 \varphi} F^{2}-\frac{20}{3} P(\varphi) .
$$

Consider solutions where $\varphi$ is constant. Equation (18) reads now

$$
e^{2 \varphi} F^{2}-2 \frac{\partial P(\varphi)}{\partial \varphi}=0
$$

and implies that $F^{2}$ is constant. Equation (45) and (19) imply then that the fivedimensional scalar curvature $\mathcal{R}$ is constant and negative. Since $F^{2}$ can be written as a product of $e^{-4 g}$ times some function of the coordinates on $L$ we deduce that both factors have to be constant. Consider $A d S_{2} \times L$ solutions. Then the above implies that $L$ is a constant curvature 3 -fold.

A solution of the form $A d S_{2} \times S^{3}$ is excluded at the level of the field equations (17) and (18). Combining the two field equations and taking $\varphi=$ const. we get for the five dimensional Ricci tensor

$$
\mathcal{R}_{\mu \nu}=\frac{4}{\bar{g}^{2}} e^{2 \varphi} \hat{R}^{-4} g_{\mu \nu}-\frac{1}{2} \bar{g}^{2} e^{\varphi} g_{\mu \nu}
$$

where $\hat{R}=e^{g}$ denotes the radius of the $S^{3}$. Note that the term in (47) proportional to $\hat{R}^{-4}$ vanishes for the components of $\mathcal{R}_{\mu \nu}$ which are not along the sphere. The time component of (47) fixes the radius of the $A d S_{2}$-part in terms of $\varphi$ for the ansatz (36) as

$$
A^{2}=\frac{2}{\bar{g}^{2}} e^{-\varphi} .
$$

On the other hand we can use (45) and (46) to express $\mathcal{R}$ in terms of $\varphi$

$$
\mathcal{R}=-\frac{3}{2} \bar{g}^{2} e^{\varphi}-\bar{g}^{2} e^{-2 \varphi} .
$$

For an $A d S_{2} \times S^{3}$ background we have $\mathcal{R}=-\frac{2}{A^{2}}+\frac{6}{\hat{R}^{2}}$, which upon using (48) leads to a contradiction.

Consider now 3-cycles based on the types $S^{2} \times E^{1}$ and $H^{2} \times E^{1}$. They too have a constant curvature and one may expect that they will give rise to $A d S_{2}$ solutions of the gauged supergravity. However, in this case the ansatz (20) does not allow for a supersymmetric solution. The reason is that from the variation of the gravitino (22) polarized along the direction of $E^{1}$ we get for the ansatz (20) one more relation between $g$ and $f$ in addition to equations (77). This prevents a solution. The nontrivial part of the spin connection of these cycles comes from the two dimensional part, i.e. $S^{2}$ or $H^{2}$. Therefore they have reduced holonomy. To implement the twist we need an abelian truncation of the $S U(2) \times U(1)$ gauged supergravity. That means that for such configurations only the 
$U(1) \subset S U(2)$ is excited. This breaks less supersymmetry compared to the cycles with $S U(2)$ holonomy. For an appropriate ansatz there are solutions of this truncated gauged supergravity which then do not correspond to D3-branes wrapped on associative 3-cycles in $G_{2}$ holonomy manifolds but rather to configurations of D3-branes wrapping around a Riemann surface $\Sigma$ in $C Y$ [6]. The details of this calculation are given in appendix A. From [14] it is clear that from the ten-dimensional point of view the abelian truncation used here coincides with the truncation used in [6] to study D3-branes wrapped around a Riemann surface in a Calabi-Yau 3-fold.

We conclude that the $\mathcal{N}=4$ five-dimensional supergravity that we are using is not capable of describing the wrapping of associative 3-cycle of the type $\Sigma \times S^{1}$. Consulting section 2.3, we see that what is missing in that context is the implementation of the $Z_{2}$ quotient which truncates the supersymmetry by a factor of two. A similar situation arises in the analysis of [12] for M5-branes wrapped around associative 3-cycles.

In section 2, we described a case (4), where the associative 3-cycle corresponds to a special Lagrangian 3-cycle. It is natural to ask whether we can argue based on the $A d S_{2} \times L$ solution for an associative 3-cycle $L$, that there should be an $A d S_{2} \times L$ solution for the corresponding special Lagrangian 3-cycle. Of course, the latter solution should preserve twice as much supercharges. For comparison we can look at the example of a Calabi-Yau 3-fold constructed as $\left(K 3 \times T^{2}\right) / \Theta[26]$, where the $\Theta$ is a freely acting involution on $K 3$ and an inversion on $T^{2}$. In this case we have supersymmetric 2-cycles (Riemann surfaces) in the Calabi-Yau 3-fold that correspond to Riemann surfaces in $K 3$. D3-branes wrapped on a Riemann surface in the Calabi-Yau 3-fold have an $A d S_{3}$ solution that preserves four supercharges. However, there is no such $A d S_{3}$ solutions that preserves eight supercharges that corresponds to D3-branes wrapped on a Riemann surface in $K 3$ [6]. Therefore it seems that we cannot draw a conclusion from the $A d S_{2}$ solutions in the associative 3 -cycle case, as to the existence of $A d S_{2}$ solutions in the special Lagrangian case.

\subsection{D3-brane wrapped on $H^{3}$}

Consider the $N$ D3-branes wrapped on an associative 3-cycle $H^{3}$. Recall that for the five dimensional metric we take the ansatz

$$
d s^{2}=e^{2 f}\left(-d t^{2}+d r^{2}\right)+e^{2 g}\left(d \phi^{2}+\sinh ^{2} \phi d \theta^{2}+\sinh ^{2} \phi \sin ^{2} \theta d \nu^{2}\right),
$$

where $f, g$ depend only on $r$. The second part of the above metric is the metric of the $H^{3}$. We have to solve (22) and (23) in this background.

The twist implies by (87) that the $S U(2)$ gauge potentials are

$$
\begin{aligned}
& A_{\theta}^{1}=a \cosh \phi, \\
& A_{\nu}^{2}=b \cos \theta, \\
& A_{\nu}^{3}=c \sin \theta \cosh \phi .
\end{aligned}
$$

In order to take advantage of the conditions (27) we write (22) more explicitly, as done in appendix B in equations (91), (92), (93) and (94). 
For the solution we impose on the field strengths (88)

$$
F_{\nu \theta}^{2} \propto \sin \theta \sinh ^{2} \phi \rightarrow \frac{a c}{b}=-\frac{1}{\bar{g}}
$$

and

$$
F_{\nu \theta}^{3}=0 \rightarrow c=-a b \bar{g} .
$$

We solve these constraints by setting

$$
a=\frac{1}{\bar{g}}, b=\frac{1}{\bar{g}}, c=-\frac{1}{\bar{g}} .
$$

From the twist we get the following constraints on the Killing spinors

$$
\begin{aligned}
\gamma_{23} \varepsilon_{a} & = \pm i\left(\Gamma_{1}\right)_{a}^{b} \varepsilon_{b} \\
\gamma_{24} \varepsilon_{a} & =\mp i\left(\Gamma_{3}\right)_{a}^{b} \varepsilon_{b} \\
\gamma_{34} \varepsilon_{a} & = \pm i\left(\Gamma_{2}\right)_{a}^{b} \varepsilon_{b}
\end{aligned}
$$

for $\left(\Gamma_{45}\right)_{a}^{b} \varepsilon_{b}= \pm i \varepsilon_{a}$ and where we have used (54).

Given the above constraints the supersymmetric variations vanish for

$$
\begin{aligned}
& g^{\prime}=-e^{f}\left\{-\sqrt{2} e^{\varphi} e^{-2 g} \frac{1}{\bar{g}}+\frac{\bar{g}}{6 \sqrt{2}}\left(2 e^{-\varphi}+e^{2 \varphi}\right)\right\}, \\
& f^{\prime}=-e^{f}\left\{\sqrt{2} e^{\varphi} e^{-2 g} \frac{1}{\bar{g}}+\frac{\bar{g}}{6 \sqrt{2}}\left(2 e^{-\varphi}+e^{2 \varphi}\right)\right\}, \\
& \varphi^{\prime}=-e^{f}\left\{\sqrt{2} e^{\varphi} e^{-2 g} \frac{1}{\bar{g}}+\frac{\bar{g}}{3 \sqrt{2}}\left(e^{-\varphi}-e^{2 \varphi}\right)\right\} .
\end{aligned}
$$

The boundary conditions as $r \rightarrow 0$ are $g(r), f(r) \rightarrow-\log (r)$ so that from (56) we get

$$
\varphi(r) \rightarrow-\frac{1}{2} r^{2} \log (r)+C_{\varphi} r^{2}
$$

As in [6] there is a relation between $g$ and $\varphi$ which reads

$$
e^{2 g+\varphi}=e^{2(g-\varphi)}+\frac{12}{\bar{g}^{2}}(g+\varphi)+C .
$$

The integration constant $C$ is related to the integration constant $C_{\varphi}(57)$ of the differential equation for $\varphi$, i.e. to the expectation value in the UV of the operator dual to $\varphi$ [6]. For an asymptotic configuration (42) we find $C=\frac{1}{4}(3-\log 4)$.

We therefore expect to have for this value of $C$ a solution interpolating between $A d S_{5}$ in the UV with $A d S_{2} \times H^{3}$ in the IR. Indeed we can find such a solution numerically. In figure (1) we plot $e^{2 f(r)}$ as obtained from a numerical solution of equations (56) when $C=\frac{1}{4}(3-\log 4)$ in comparison to the expected behaviour in the IR, $e^{2 f}=\frac{1}{4^{\frac{4}{3}} r^{2}}(42)$. We see that indeed they coincide in the IR. 


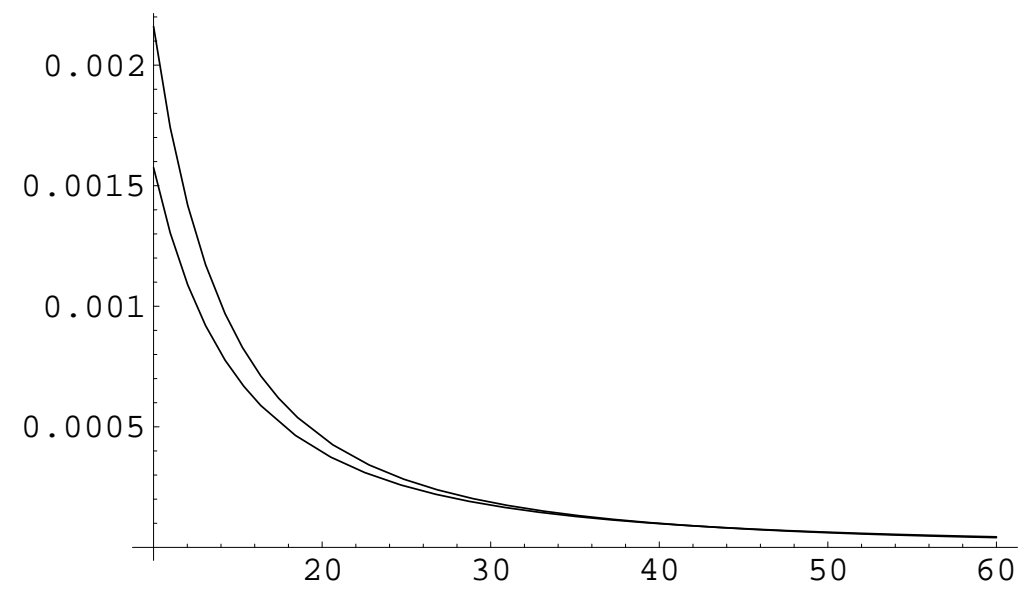

Figure 1: In this figure we plot $e^{2 f(r)}$ as obtained from a numerical solution of equations (56) when $C=\frac{1}{4}(3-\log 4)$ in comparison to the expected behaviour in the IR, $e^{2 f}=\frac{1}{4^{\frac{4}{3}} r^{2}}$ in equation (42). We see that the two coincide in the IR.

In figure (2) we plot $e^{2 g(r)}$ as obtained from a numerical solution in comparison to the expected behaviour in the IR, $e^{2 g}=4^{-\frac{1}{3}}(42)$. Again we see that they coincide in the IR.

An expansion of (58) leads to $C_{\varphi}=\frac{C}{3}$ so that

$$
C_{\varphi}^{c r i t .}=\frac{1}{4}-\frac{\log 4}{12}
$$

As noted, we can interpret $C_{\varphi}$ in (57) roughly as the expectation value of the operator dual to $\varphi . C_{\varphi}=C_{\varphi}^{c r i t .}$ is then the value for which the Higgs and Coulomb branches "intersect" and this is where we expect a fixed point. For $C_{\varphi}>C_{\varphi}^{c r i t}$. we move into the Coulomb branch while for $C_{\varphi}<C_{\varphi}^{c r i t}$. we move into the Higgs branch. It is then of interest to see what happens to the supergravity solution as we vary $C_{\varphi}$, which enters as a boundary value for $\varphi$. This analysis can be done numerically.

The numerical analysis of (56) shows singular solutions for $C_{\varphi}>C_{\varphi}^{\text {crit. }}$, as depicted in figure (3) and (4).

We can check the nature of the singularity according to the criterion of [27]. We need to compute the effective potential of the five-dimensional gauged supergravity corresponding to the above solution. For our ansatz with the gauge field determined by the twist we find from the Lagrangian given in [13]

$$
V_{\text {eff }}=\frac{3}{16} e^{2 \varphi} e^{-4 g}-\left(e^{-2 \varphi}+2 e^{\varphi}\right) .
$$

Since this potential diverges near the singularity it is of "bad" type. The meaning of this singularity is that in the limit that we consider the Higgs and the Coulomb branches 


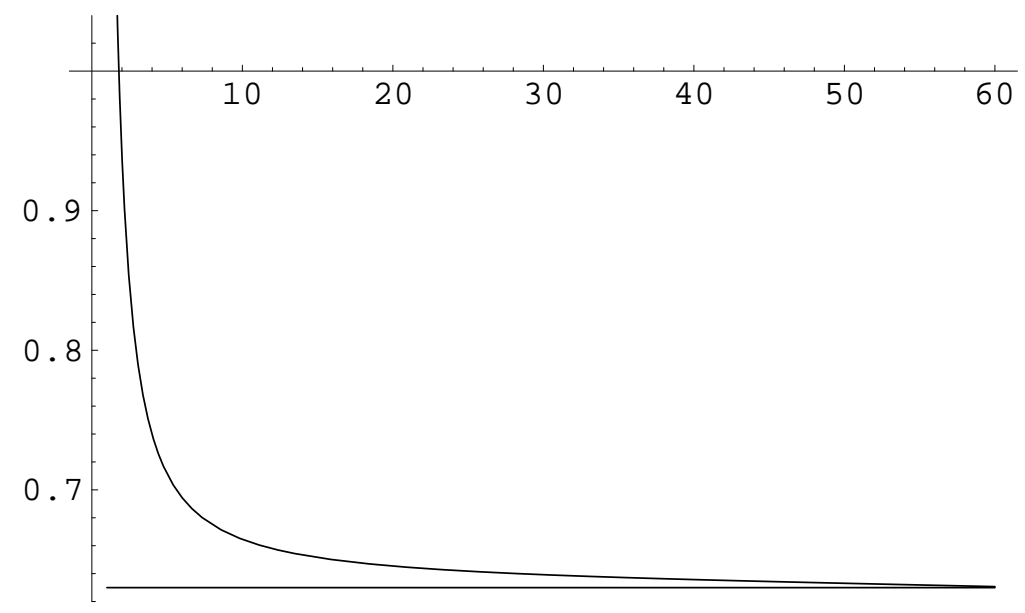

Figure 2: In this figure we plot $e^{2 g(r)}$ as obtained from a numerical solution in comparison to the expected behaviour in the IR, $e^{2 g}=4^{-\frac{1}{3}}$ in equation (42). We see that the two coincide in the IR.

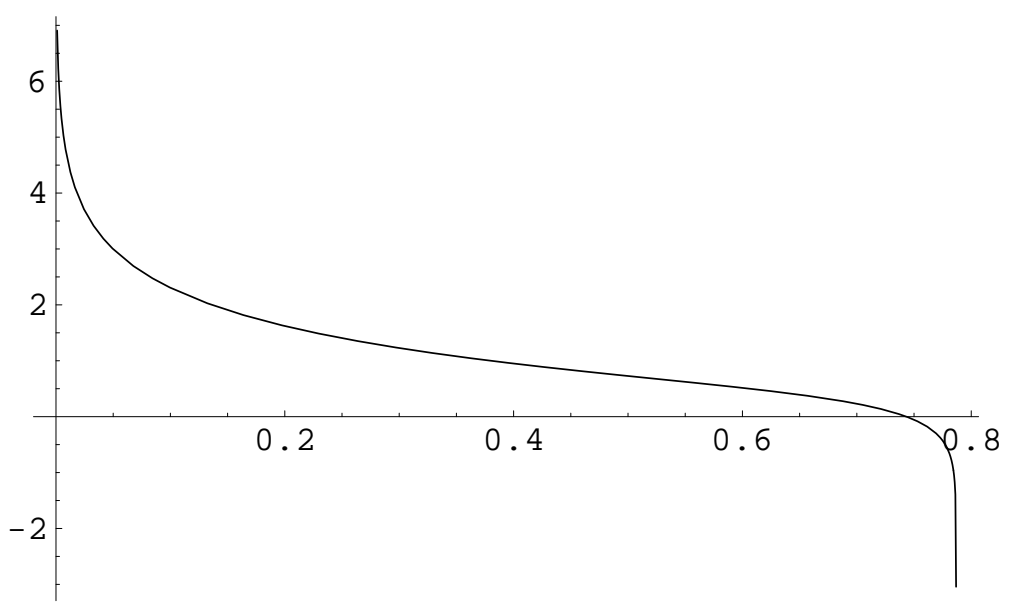

Figure 3: The numerical behaviour of $g(r)$ approaching a "bad" singularity in the Coulomb branch, $C_{\varphi}>C_{\varphi}^{\text {crit. }}$ 
decouple, and we remain at low-energy with a $(0+1)$-dimensional $\sigma$-model on the Higgs branch.

The numerical solution for $C_{\varphi}<C_{\varphi}^{c r i t}$. has a different behaviour. The radii $\exp (2 f(r))$ and $\exp (2 g(r))$ are shrinking in the IR as seen in figures (5) and (6), which means increasing of the curvature of the solution. However, in this case the behaviour of $\varphi$ is different as seen in figure (7) and the singularity in the extreme IR is of a "good" type. 


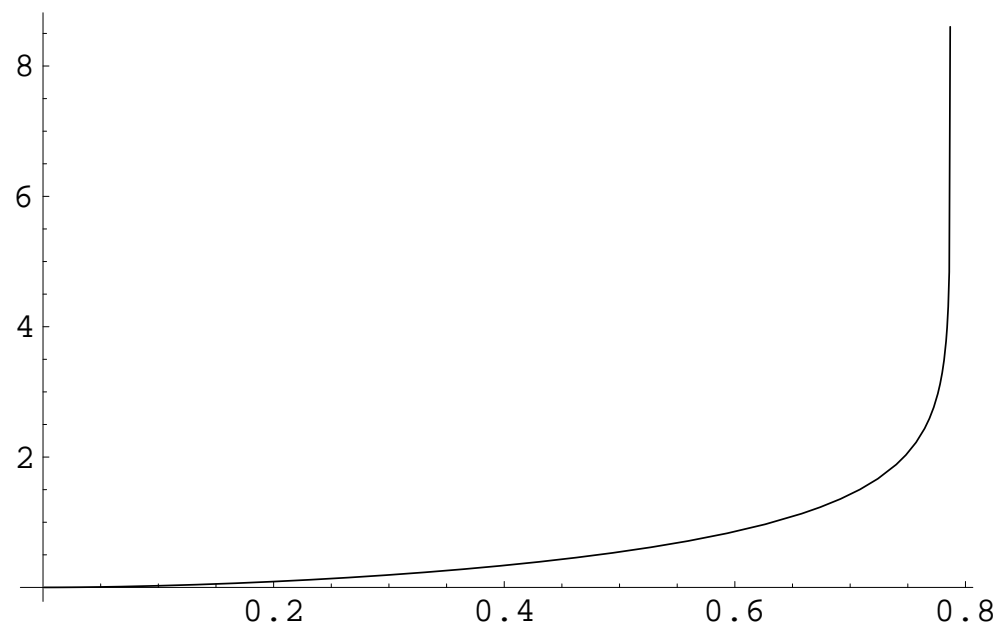

Figure 4: $\varphi(r)$ blows up near a "bad" singularity in the Coulomb branch, $C_{\varphi}>C_{\varphi}^{\text {crit. }}$

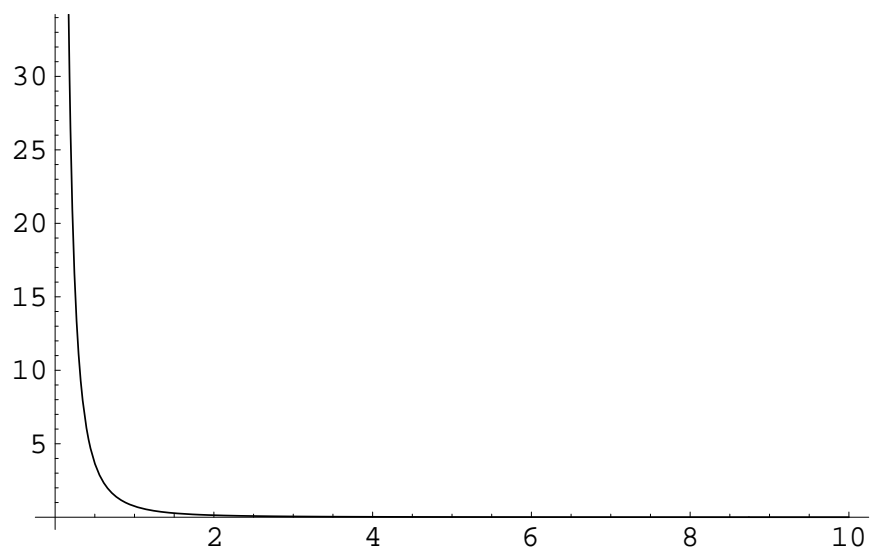

Figure 5: The radius $e^{2 f(r)}$ is shrinking in the IR, in the Higgs branch $C_{\varphi}<C_{\varphi}^{c r i t}$. However the effective potential is bounded from above and the singularity is of a "good" type. 


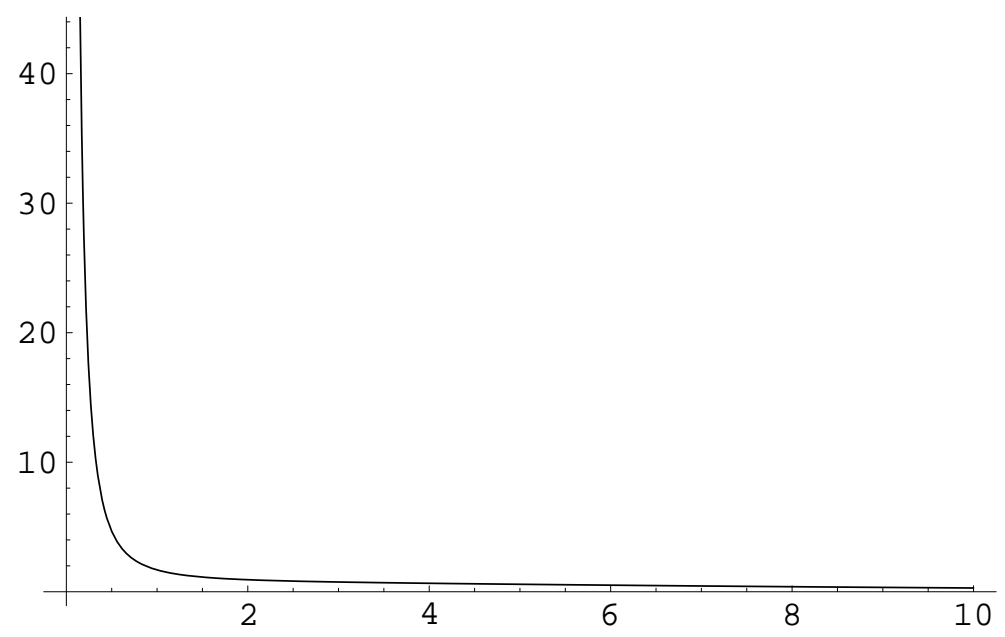

Figure 6: The qualitative behaviour of the radius $e^{2 g(r)}$ is very similar to that of $e^{2 f(r)}$ in the Higgs branch, $C_{\varphi}<C_{\varphi}^{c r i t .}$. It is also shrinking in the IR.

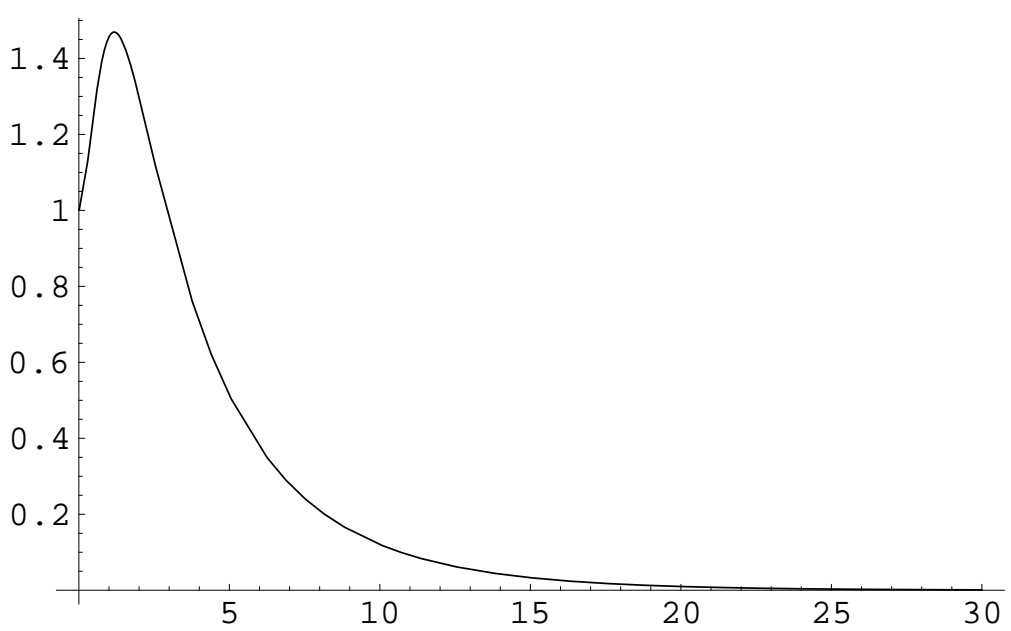

Figure 7: $e^{2 \varphi(r)}$ in the Higgs branch, $C_{\varphi}<C_{\varphi}^{c r i t}$. It goes to zero as it approaches the "good" singularity in the IR. 


\subsection{D3-brane wrapped on $S^{3}$}

Consider now the $N D$ 3-branes wrapped on an associative 3-cycle $S^{3}$. Recall that for the five dimensional metric we take the ansatz

$$
d s^{2}=e^{2 f}\left(-d t^{2}+d r^{2}\right)+e^{2 g}\left(d \phi^{2}+\sin ^{2} \phi d \theta^{2}+\sin ^{2} \phi \sin ^{2} \theta d \nu^{2}\right),
$$

where $f, g$ depend only on $r$.

The supergravity equations can be obtained from (56). In (56), due to the twist the first term is proportional to the scalar curvature of the supersymmetric cycle. The transition $H^{3} \rightarrow S^{3}$ thus induces a minus sign in this term. This can also be seen via the coordinate transformation $\phi \rightarrow i \phi$ as argued in [6]. Hence we get the following equations for the $S^{3}$ case

$$
\begin{aligned}
& g^{\prime}=-e^{f}\left\{\sqrt{2} e^{\varphi} e^{-2 g} \frac{1}{\bar{g}}+\frac{\bar{g}}{6 \sqrt{2}}\left(2 e^{-\varphi}+e^{2 \varphi}\right)\right\}, \\
& f^{\prime}=-e^{f}\left\{-\sqrt{2} e^{\varphi} e^{-2 g} \frac{1}{\bar{g}}+\frac{\bar{g}}{6 \sqrt{2}}\left(2 e^{-\varphi}+e^{2 \varphi}\right)\right\}, \\
& \varphi^{\prime}=-e^{f}\left\{-\sqrt{2} e^{\varphi} e^{-2 g} \frac{1}{\bar{g}}+\frac{\bar{g}}{3 \sqrt{2}}\left(e^{-\varphi}-e^{2 \varphi}\right)\right\} .
\end{aligned}
$$

Again, the boundary conditions as $r \rightarrow 0$ are $g(r), f(r) \rightarrow-\log (r)$ so that from (62) we get

$$
\varphi(r) \rightarrow \frac{1}{2} r^{2} \log (r)+C_{\varphi} r^{2}
$$

We can find a numerical solution as depicted in figures (8) and (9). The numerical solution has the same behaviour independently of the value of $C_{\varphi}$. The singular behaviour is as for the $H^{3}$ case on the Coulomb branch, i.e. the singularity is of the "bad" type.

As before, the "bad" singularity in the Coulomb branch can be explained by the fact that in the limit that we consider the Higgs and the Coulomb branches decouple, and we remain at low-energy with a $(0+1)$-dimensional $\sigma$-model on the Higgs branch. However, in this case, unlike $H^{3}$, we do not expect a Higgs branch for the dual theory since there are no harmonic spinors on $S^{3}$ and therefore it is rigid in the ambient seven-dimensional manifold. This explains the difference between the type of singularity along the Higgs branch in $H^{3}$ compared to $S^{3}$. 


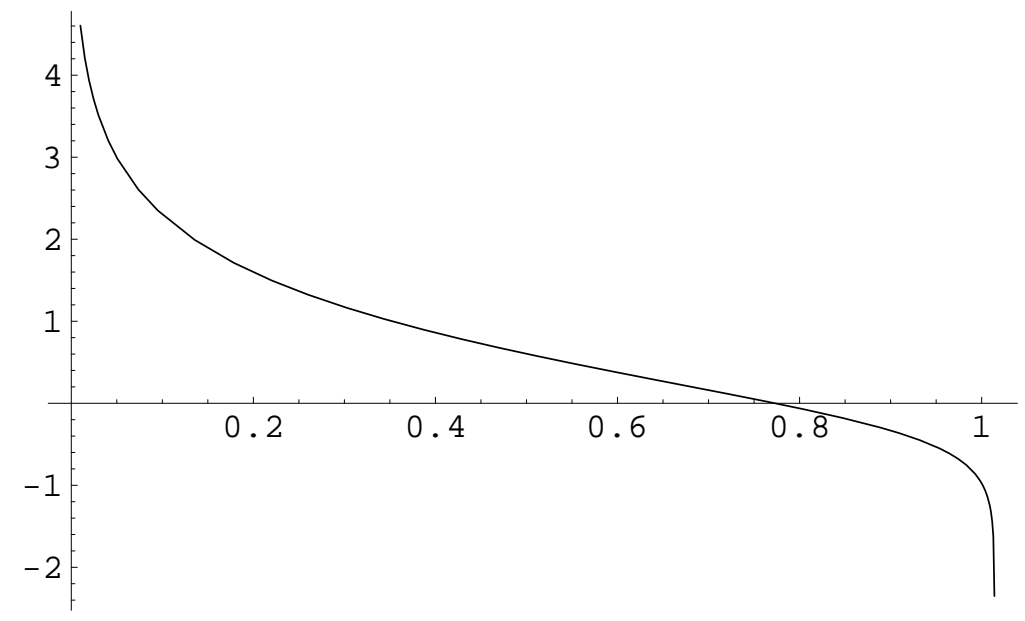

Figure 8: A numerical solution for $g(r)$ in the case of $S^{3}$ cycle.

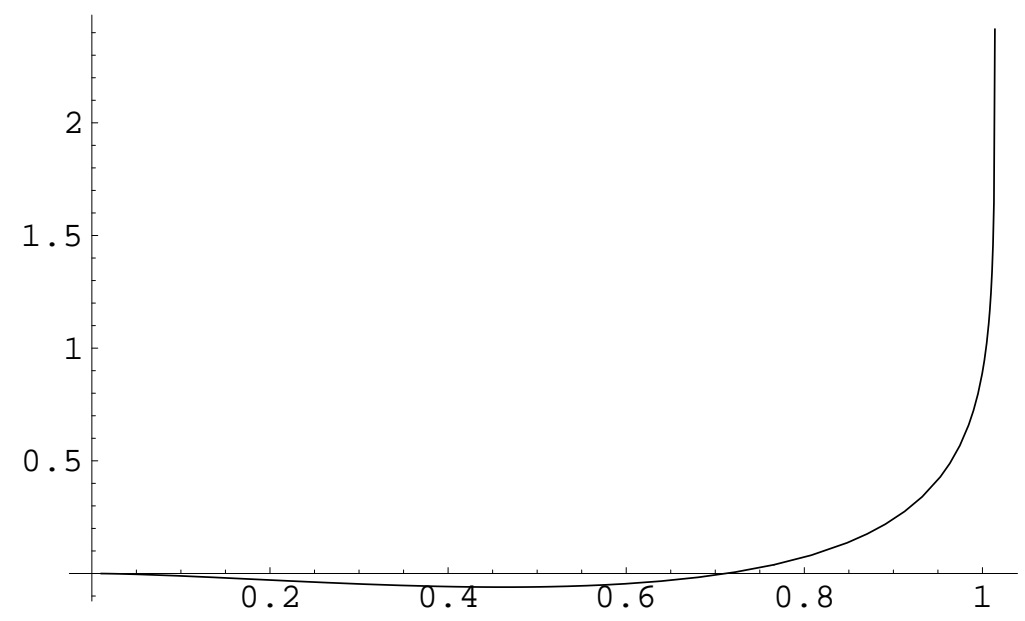

Figure 9: A numerical solution of $\varphi(r)$ for $S^{3}$ cycle. 
Acknowledgement: We are grateful to J. Maldacena for pointing out that the correct interpretation of the supergravity solutions in section 4 is that of D3-branes wrapping associative 3-cycles and not special Lagrangian 3-cycles as written in the previous version of this paper. The $A d S_{2} \times d s_{H^{3}}^{2}$ solution in section 4 has been independently obtained by J. Maldacena and C. Nunez. We would like to thank J. Maldacena for this information and for other valuable discussions. 


\section{Appendix}

\section{A The Riemann surface case}

For the case of a D3-brane wrapped around a Riemann surface we get from (9) and (10) the solution of [6]. The metric ansatz reads

$$
d s^{2}=e^{2 f}\left(d r^{2}+d z^{2}-d t^{2}\right)+\frac{e^{2 g}}{y^{2}}\left(d x^{2}+d y^{2}\right)
$$

and the corresponding spin connection

$$
\begin{aligned}
& \omega_{x}^{34}=-\frac{1}{y}, \\
& \omega_{x}^{31}=\omega_{y}^{41}=\frac{e^{g-f} g^{\prime}}{y}, \\
& \omega_{z}^{21}=\omega_{t}^{01}=f^{\prime},
\end{aligned}
$$

where the flat indices $(1,2, .$.$) are connected with the curved ones by the vielbein$

$$
\begin{aligned}
& e_{t}^{0}=e_{r}^{1}=e_{z}^{2}=e^{f}, \\
& e_{x}^{3}=e_{y}^{4}=\frac{e^{g}}{y}
\end{aligned}
$$

We set $B_{\mu \nu}^{\alpha}$ to zero and take $\Phi$ to depend only on $r$, furthermore we truncate the $S U(2) \times$ $U(1)$ gauge group to the Cartan subgroup $U(1) \times U(1)$. From the spin connection (65) we get the gauge potentials

$$
a_{x}=\frac{a}{y}, A_{x}^{3}=\frac{b}{y}
$$

and the corresponding field strengths

$$
f_{x y}=\frac{a}{y^{2}}, F_{x y}^{3}=\frac{b}{y^{2}} .
$$

In addition we impose the following conditions on the spinors

$$
\gamma_{34} \varepsilon_{a}=-i \varepsilon_{a}, \gamma_{1} \varepsilon_{a}=\varepsilon_{a},
$$

as in [6]. We rescale the coupling constants [13], $g_{1}=\frac{\bar{g}}{\sqrt{2}}$ and $g_{2}=\bar{g}$. In this background with the above definitions the susy-variation of the $x$-component of the gravitino reads

$$
\begin{aligned}
& \delta \psi_{x a}=\partial_{x} \varepsilon_{a}+\frac{1}{2} \omega_{x}^{34} \gamma_{34} \varepsilon_{a}+\frac{1}{2} \omega_{x}^{31} \gamma_{31} \varepsilon_{a}+\frac{1}{2} \frac{\bar{g}}{2} \frac{a}{y}\left(\Gamma_{45}\right)_{a}^{b} \varepsilon_{b}+ \\
& +\frac{1}{2} \bar{g} \frac{b}{y}\left(\Gamma_{345}\right)_{a}^{b} \varepsilon_{b}-i \gamma_{x} \frac{1}{12 \sqrt{2}} \bar{g}\left(2 e^{-\varphi}+e^{2 \varphi}\right)\left(\Gamma_{45}\right)_{a}^{b} \varepsilon_{b}+ \\
& +i \frac{2}{3} \sqrt{\frac{1}{2}} \gamma^{y} e^{\varphi} F_{x y}^{3}\left(\Gamma_{3}\right)_{a}^{b} \varepsilon_{b}+i \frac{1}{3} e^{-2 \varphi} f_{x y} \gamma^{y} \varepsilon_{a},
\end{aligned}
$$


where we have set $\varphi \equiv \sqrt{\frac{2}{3}} \phi$. Setting (70) to zero yields

$$
\bar{g}\left(\frac{a}{\sqrt{2}} \Gamma_{45}+b \Gamma_{345}\right)_{a}^{b} \varepsilon_{b}=-i \varepsilon_{a}
$$

and

$$
\begin{gathered}
g^{\prime} \varepsilon_{a}=e^{f}\left(\frac{i}{6 \sqrt{2}} \bar{g}\left(2 e^{-\varphi}+e^{2 \varphi}\right)\left(\Gamma_{45}\right)_{a}^{b} \varepsilon_{b}-\right. \\
\left.-\frac{4}{3} \sqrt{\frac{1}{2}} e^{-2 g} e^{\varphi} b\left(\Gamma_{3}\right)_{a}^{b} \varepsilon_{b}-\frac{2}{3} e^{-2 g} e^{-2 \varphi} a \varepsilon_{a}\right)
\end{gathered}
$$

Eqn. (71) can be solved in the following ways

$$
\begin{aligned}
& \left(\Gamma_{45}\right)_{a}^{b} \varepsilon_{b}=i \varepsilon_{a},\left(\Gamma_{3}\right)_{a}^{b} \varepsilon_{b}= \pm \varepsilon_{a} \rightarrow \bar{g}\left(\frac{a}{\sqrt{2}} \pm b\right)=-1, \\
& \left(\Gamma_{45}\right)_{a}^{b} \varepsilon_{b}=-i \varepsilon_{a},\left(\Gamma_{3}\right)_{a}^{b} \varepsilon_{b}= \pm \varepsilon_{a} \rightarrow \bar{g}\left(\frac{a}{\sqrt{2}} \pm b\right)=1,
\end{aligned}
$$

Solutions which differ in the eigenvalue of $\Gamma_{3}$ are equivalent whereas solutions with different eigenvalues of $\Gamma_{45}$ give different solutions. Using (73) we can solve (72) by choosing $b=0, a=-\frac{\sqrt{2}}{\bar{g}}$ to give

$$
g^{\prime}=e^{f}\left(-\frac{\bar{g}}{6 \sqrt{2}}\left(2 e^{-\varphi}+e^{2 \varphi}\right)+\frac{2}{3} \frac{\sqrt{2}}{\bar{g}} e^{-2 g} e^{-2 \varphi}\right),
$$

which is part of the solution for the twist preserving $(4,4)$ supersymmetry in [6]. Exact correspondence is obtained if we set $\bar{g}=2 \sqrt{2}$.

If we set $a=0, b=-\frac{1}{\bar{g}}$ we get the corresponding expression for the twist preserving $(2,2)$ supersymmetry

$$
g^{\prime}=e^{f}\left(-\frac{1}{3}\left(2 e^{-\varphi}+e^{2 \varphi}\right)+\frac{1}{3} e^{-2 g} e^{\varphi}\right) .
$$

The solutions corresponding to (74) give an overall sign in (75) and (76) and will be ruled out by the boundary conditions for $f, g$ in the limit $r \rightarrow 0$. By solving the remaining fermionic supersymmetry variations we get the differential equations for $f$ and $\varphi$.

The case $H^{2}$ resembles $H^{3}$ while the $S^{2}$ case resembles the $S^{3}$ one. In the $H^{2}$ case the equations read [6]

$$
\begin{aligned}
g^{\prime} & =e^{f}\left(-\frac{1}{3}\left(2 e^{-\varphi}+e^{2 \varphi}\right)+\frac{1}{3} e^{-2 g} e^{\varphi}\right), \\
f^{\prime} & =-\frac{e^{f}}{6}\left(2\left(2 e^{-\varphi}+e^{2 \varphi}\right)+e^{-2 g} e^{\varphi}\right) \\
\varphi^{\prime} & =\frac{2}{3} e^{f}\left(\left(-e^{-\varphi}+e^{2 \varphi}\right)-\frac{1}{4} e^{-2 g} e^{\varphi}\right) .
\end{aligned}
$$


The boundary conditions as $r \rightarrow 0$ are $g(r), f(r) \rightarrow-\log (r)$ so that from (77) we get

$$
\varphi(r) \rightarrow-\frac{1}{6} r^{2} \log (r)+C_{\varphi} r^{2} .
$$

There is a relation between $g$ and $\varphi$ which reads

$$
e^{2 g+\varphi}=e^{2(g-\varphi)}+\frac{1}{2}(g+2 \varphi)+C .
$$

The $A d S_{3} \times H^{2}$ solution of (77) gives $C=\frac{1}{4}$.

We therefore expect to have for this value of $C$ a solution interpolating between $A d S_{5}$ in the UV with $A d S_{3} \times H^{2}$ in the IR and indeed we find one. Similar solutions were constructed in [28] in the context of $D=5, N=2$ gauged supergravity which were generalized in [29] where also an analysis of solutions of the form $M_{3} \times M_{2}$ was given.

An expansion of (79) leads to $C_{\varphi}=\frac{C}{3}$ so that

$$
C_{\varphi}^{c r i t .}=\frac{1}{12} .
$$

Again, one interprets $C_{\varphi}$ in (78) roughly as the expectation value of the operator dual to $\varphi . C_{\varphi}=C_{\varphi}^{\text {crit. }}$ is then the value for which the Higgs and Coulomb branches "intersect" and this is where we expect a fixed point. For $C_{\varphi}>C_{\varphi}^{\text {crit. }}$ we move into the Coulomb branch while for $C_{\varphi}<C_{\varphi}^{c r i t .}$ we move into the Higgs branch. It is then of interest to see what happens to the supergravity solution as we vary $C_{\varphi}$, which enters as a boundary value for $\varphi$. The numerical analysis of (77) shows singular solutions in both cases. However, the nature of the singularity is different and like the $H^{3}$ case it is of the "good" type along the Higgs branch and of the "bad" type along the Coulomb branch, i.e. the potential

$$
V_{e f f}=\frac{1}{4} e^{2 \varphi} e^{-4 g}-4\left(e^{-2 \varphi}+2 e^{\varphi}\right) .
$$

is unbounded from above near the singularity.

In the case of $S^{2}$ we find, like in the $S^{3}$ case, that the singularity is of the "bad" type independently of the value of $C_{\varphi}$.

As before, the "bad" singularity in the Coulomb branch can be explained by the fact that in the limit that we consider the Higgs and the Coulomb branches decouple, and

we remain at low-energy with a $(1+1)$-dimensional $\sigma$-model on the Higgs branch. The "bad" singularity on the Higgs branch in the $S^{2}$ case, indicates its absence.

\section{B The $S^{3}, H^{3}$ examples}

For the five dimensional metric we take the ansatz

$$
d s^{2}=e^{2 f}\left(-d t^{2}+d r^{2}\right)+e^{2 g}\left(d \phi^{2}+\sin ^{2} \phi d \theta^{2}+\sin ^{2} \phi \sin ^{2} \theta d \nu^{2}\right),
$$

where $f, g$ depend only on $r$. The second part of the above metric is the metric of the $S^{3}$. 
The components of the vielbein of this metric read

$$
\begin{aligned}
& e_{t}^{0}=e^{f}, \\
& e_{r}^{1}=e^{f}, \\
& e_{\phi}^{2}=e^{g}, \\
& e_{\theta}^{3}=e^{g} \sin \phi, \\
& e_{\nu}^{4}=e^{g} \sin \phi \sin \theta .
\end{aligned}
$$

For the boundary conditions to be imposed on the $S U(2)$ gauge potentials we need the spin connection on the $S^{3}$. For the $S^{3}$ part of the above metric we get the following nonvanishing connection coefficients

$$
\begin{aligned}
& \Gamma_{\theta \theta}^{\phi}=-\sin \phi \cos \phi, \\
& \Gamma_{\theta \phi}^{\theta}=\cot \phi, \\
& \Gamma_{\nu \nu}^{\phi}=-\sin ^{2} \theta \sin \phi \cos \phi, \\
& \Gamma_{\nu \phi}^{\nu}=\cot \phi, \\
& \Gamma_{\nu \nu}^{\theta}=-\sin \theta \cos \theta, \\
& \Gamma_{\nu \theta}^{\nu}=\cot \theta .
\end{aligned}
$$

With the vielbeins

$$
\begin{aligned}
e_{\phi}^{\overline{1}} & =1, \\
e_{\theta}^{\overline{2}} & =\sin \phi, \\
e_{\nu}^{\overline{3}} & =\sin \phi \sin \theta,
\end{aligned}
$$

we get for the spin connection

$$
\begin{aligned}
& \omega_{\theta}^{\overline{1} \overline{2}}=-\cos \phi=\omega_{\theta}^{23}, \\
& \omega_{\nu}^{1 \overline{3}}=-\sin \theta \cos \phi=\omega_{\nu}^{24}, \\
& \omega_{\nu}^{\overline{2} \overline{3}}=-\cos \theta=\omega_{\nu}^{34},
\end{aligned}
$$

where the barred superscripts denote flat indices on $S^{3}$. Thus we are led to the gauge potentials

$$
\begin{aligned}
A_{\theta}^{1} & =a \cos \phi, \\
A_{\nu}^{2} & =b \cos \theta, \\
A_{\nu}^{3} & =c \sin \theta \cos \phi,
\end{aligned}
$$

(identifying $23 \leftrightarrow 1,34 \leftrightarrow 2,24 \leftrightarrow 3$ ). The only nonvanishing components of the corresponding field strength are

$$
\begin{aligned}
& F_{\theta \phi}^{1}=a \sin \phi, \\
& F_{\nu \theta}^{2}=\sin \theta\left(b+\bar{g} a c \cos ^{2} \phi\right), \\
& F_{\nu \phi}^{3}=c \sin \theta \sin \phi, \\
& F_{\nu \theta}^{3}=-\cos \theta \cos \phi(c+\bar{g} a b),
\end{aligned}
$$


where the superscripts refer to the generators $T_{a}$ of $S U(2)$, which is suitably embedded in the $\operatorname{Spin}(5) R$-symmetry group, with the Lie algebra $\left[T_{a}, T_{b}\right]=\epsilon_{a b c} T_{c}$. The coupling constant $\bar{g}$ is included for later reference. The other nonvanishing connection coefficients for the metric (82) are

$$
\begin{aligned}
& \Gamma_{t t}^{r}=f^{\prime}, \\
& \Gamma_{t r}^{t}=f^{\prime}, \\
& \Gamma_{\phi \phi}^{r}=-e^{2(g-f)} g^{\prime}, \\
& \Gamma_{\theta \theta}^{r}=-e^{2(g-f)} g^{\prime} \sin ^{2} \phi, \\
& \Gamma_{\nu \nu}^{r}=-e^{2(g-f)} g^{\prime} \sin ^{2} \phi \sin ^{2} \theta, \\
& \Gamma_{\phi r}^{\phi}=\Gamma_{\theta r}^{\theta}=\Gamma_{\nu r}^{\nu}=g^{\prime}, \\
& \Gamma_{r r}^{r}=f^{\prime},
\end{aligned}
$$

while calculating the spin connection yields

$$
\begin{aligned}
& \omega_{t}^{10}=-f^{\prime}, \\
& \omega_{\phi}^{12}=-e^{g-f} g^{\prime}, \\
& \omega_{\theta}^{13}=-e^{g-f} g^{\prime} \sin \phi, \\
& \omega_{\nu}^{14}=-e^{g-f} g^{\prime} \sin \phi \sin \theta .
\end{aligned}
$$

The variation of the fermionic fields is given by

$$
\begin{aligned}
& \delta \psi_{t a}= \\
& =\partial_{t} \varepsilon_{a}+\frac{1}{2} f^{\prime} \gamma_{0} \varepsilon_{a}-i e^{f} \gamma_{0} \frac{1}{12 \sqrt{2}} \bar{g}\left(2 e^{-\varphi}+e^{2 \varphi}\right)\left(\Gamma_{45}\right)_{a}^{b} \varepsilon_{b}+ \\
& +i \frac{1}{3} \sqrt{\frac{1}{2}} e^{\varphi} e^{(f-2 g)} \sin ^{-1} \phi\left\{\gamma_{023} F_{\theta \phi}^{1}\left(\Gamma_{1}\right)_{a}^{b} \varepsilon_{b}+\right. \\
& \left.+\sin ^{-1} \phi \sin ^{-1} \theta \gamma_{034}\left(F_{\nu \theta}^{2}\left(\Gamma_{2}\right)_{a}^{b} \varepsilon_{b}+F_{\nu \theta}^{3}\left(\Gamma_{3}\right)_{a}^{b} \varepsilon_{b}\right)+\sin ^{-1} \theta \gamma_{024} F_{\nu \phi}^{3}\left(\Gamma_{3}\right)_{a}^{b} \varepsilon_{b}\right\}=0 \\
& \quad \delta \psi_{\phi a}= \\
& =\partial_{\phi} \varepsilon_{a}+\frac{1}{2} e^{g-f} g^{\prime} \gamma_{2} \varepsilon_{a}-i e^{g} \gamma_{2} \frac{1}{12 \sqrt{2}} \bar{g}\left(2 e^{-\varphi}+e^{2 \varphi}\right)\left(\Gamma_{45}\right)_{a}^{b} \varepsilon_{b}+ \\
& \quad+i \frac{1}{3} \sqrt{\frac{1}{2}} e^{\varphi} e^{-g} \sin ^{-2} \phi \sin ^{-1} \theta \gamma_{234}\left(F_{\nu \theta}^{2}\left(\Gamma_{2}\right)_{a}^{b} \varepsilon_{b}+F_{\nu \theta}^{3}\left(\Gamma_{3}\right)_{a}^{b} \varepsilon_{b}\right)+ \\
& \quad+i \frac{2}{3} \sqrt{\frac{1}{2}} e^{\varphi} e^{-g} \sin ^{-1} \phi\left(\sin ^{-1} \theta \gamma_{4} F_{\phi \nu}^{3}\left(\Gamma_{3}\right)_{a}^{b} \varepsilon_{b}+\gamma_{3} F_{\phi \theta}^{1}\left(\Gamma_{1}\right)_{a}^{b} \varepsilon_{b}\right)=0
\end{aligned}
$$

$\delta \psi_{\theta a}=$ 


$$
\begin{aligned}
& =\partial_{\theta} \varepsilon_{a}-\frac{1}{2} \cos \phi \gamma_{23} \varepsilon_{a}+\frac{1}{2} e^{g-f} g^{\prime} \sin \phi \gamma_{3} \varepsilon_{a}+\frac{1}{2} \bar{g} a \cos \phi\left(\Gamma_{145}\right)_{a}^{b} \varepsilon_{b}- \\
& -i e^{g} \sin \phi \gamma_{3} \frac{1}{12 \sqrt{2}} \bar{g}\left(2 e^{-\varphi}+e^{2 \varphi}\right)\left(\Gamma_{45}\right)_{a}^{b} \varepsilon_{b}-i \frac{1}{3} \sqrt{\frac{1}{2}} e^{\varphi} e^{-g} \sin ^{-1} \theta \gamma_{234} F_{\nu \phi}^{3}\left(\Gamma_{3}\right)_{a}^{b} \varepsilon_{b}+ \\
& +i \frac{2}{3} \sqrt{\frac{1}{2}} e^{\varphi} e^{-g}\left\{\gamma_{2} F_{\theta \phi}^{1}\left(\Gamma_{1}\right)_{a}^{b} \varepsilon_{b}+\sin ^{-1} \phi \sin ^{-1} \theta \gamma_{4}\left(F_{\theta \nu}^{2}\left(\Gamma_{2}\right)_{a}^{b} \varepsilon_{b}+F_{\theta \nu}^{3}\left(\Gamma_{3}\right)_{a}^{b} \varepsilon_{b}\right)\right\}=0, \\
& \delta \psi_{\nu a}= \\
& =\partial_{\nu} \varepsilon_{a}-\frac{1}{2} \sin \theta \cos \phi \gamma_{24} \varepsilon_{a}-\frac{1}{2} \cos \theta \gamma_{34} \varepsilon_{a}+\frac{1}{2} e^{g-f} g^{\prime} \sin \phi \sin \theta \gamma_{4} \varepsilon_{a}+ \\
& +\frac{1}{2} \bar{g} b \cos \theta\left(\Gamma_{245}\right)_{a}^{b} \varepsilon_{b}+\frac{1}{2} \bar{g} c \sin \theta \cos \phi\left(\Gamma_{345}\right)_{a}^{b} \varepsilon_{b}- \\
& -i e^{g} \sin \phi \sin \theta \gamma_{4} \frac{1}{12 \sqrt{2}} \bar{g}\left(2 e^{-\varphi}+e^{2 \varphi}\right)\left(\Gamma_{45}\right)_{a}^{b} \varepsilon_{b}+i \frac{1}{3} \sqrt{\frac{1}{2}} e^{\varphi} e^{-g} \sin \theta \gamma_{234} F_{\theta \phi}^{1}\left(\Gamma_{1}\right)_{a}^{b} \varepsilon_{b}+ \\
& +i \frac{2}{3} \sqrt{\frac{1}{2}} e^{\varphi} e^{-g}\left\{\sin ^{-1} \phi \gamma_{3}\left(F_{\nu \theta}^{2}\left(\Gamma_{2}\right)_{a}^{b} \varepsilon_{b}+F_{\nu \theta}^{3}\left(\Gamma_{3}\right)_{a}^{b} \varepsilon_{b}\right)+\gamma_{2} F_{\nu \phi}^{3}\left(\Gamma_{3}\right)_{a}^{b} \varepsilon_{b}\right\}=0 .
\end{aligned}
$$

The $H^{3}$ case is obtained from the above by $\sin (\phi) \rightarrow \sinh (\phi)$. 


\section{References}

[1] K. Becker, M. Becker and A. Strominger, "Fivebranes, Membranes and NonPerturbative String Theory", Nucl.Phys. B456 (1995) 130, hep-th/9507158.

[2] M. Bershadsky, V. Sadov and C. Vafa, "D-Branes and Topological Field Theories", Nucl.Phys. B463 (1996) 420, hep-th/9511222.

[3] H. Ooguri, Y. Oz and Z. Yin, "D-Branes on Calabi-Yau Spaces and Their Mirrors", Nucl.Phys. B477 (1996) 407, hep-th/9606112.

[4] K.Becker, M.Becker, D.R.Morrison, H.Ooguri, Y.Oz and Z.Yin, "Supersymmetric Cycles in Exceptional Holonomy Manifolds and Calabi-Yau 4-Folds", Nucl.Phys. B480 (1996) 225, hep-th/9608116.

[5] S. Kachru, "Lectures on Warped Compactifications and Stringy Brane Constructions", hep-th/0009247.

[6] J. Maldacena and C.Nunez, "Supergravity description of field theories on curved manifolds", hep-th/0007018.

[7] M. Alishahiha and Y. Oz, "AdS/CFT and BPS Strings in Four Dimensions", Phys.Lett. B465 (1999) 136.

[8] M. Cvetic, H. Lu, C.N. Pope, J.F. Vazquez-Poritz, "AdS in Warped Spacetimes", hep-th/0005246.

[9] A. Fayyazuddin and D. J. Smith, "Localized intersections of M5-branes and fourdimensional superconformal field theories", JHEP 9904 (1999) 030, hep-th/9902210; "Warped AdS near-horizon geometry of completely localized intersections of M5branes", JHEP 0010 (2000) 023, hep-th/0006060; B. Brinne, A. Fayyazuddin, S. Mukhopadhyay, D. J. Smith, "Supergravity M5-branes wrapped on Riemann surfaces and their QFT duals", hep-th/0009047.

[10] J. Maldacena and C.Nunez, "Towards the large N limit of pure N=1 super Yang Mills", hep-th/0008001.

[11] I. Klebanov and M. Strassler, "Supergravity and a Confining Gauge Theory: Duality Cascades and $\chi$ SB-Resolution of Naked Singularities", JHEP 0008 (2000) 052. hepth/0007191.

[12] B. Acharya, J. Gauntlett and N. Kim, "Fivebranes Wrapped On Associative ThreeCycles", hep-th/0011190.

[13] L. J. Romans, "Gauged $N=4$ supergravities in five dimensions and their magnetovac backgrounds", Nucl. Phys. B267 (1986) 433. 
[14] H. Lu, C. N. Pope and T. Tran, "Five-dimensional $N=4, S U(2) \times U(1)$ gauged supergravities from type IIB", hep-th/9909203.

[15] W. Thurston, Bull. Am. Math. Soc. (N.S.) 6 (1982) 357.

[16] R. Harvey and H. B. Lawson, "Calibrated Geometries", Acta. Math. 148 (1982) 47.

[17] R. C. McLean, "Deformations of calibrated submanifolds", Comm. Anal. Geom. 6 (1998) 705-747.

[18] O. Aharony, M. Berkooz, S. Kachru, N. Seiberg and E. Silverstein, "Matrix Description of Interacting Theories in Six Dimensions", Adv.Theor.Math.Phys. 1 (1998) 148. hep-th/9707079.

[19] E. Witten, "On The Conformal Field Theory Of The Higgs Branch", JHEP 9707 (1997) 003, hep-th/9707093.

[20] S. Kachru, Y. Oz and Z. Yin, "Matrix Description of Intersecting M5 Branes", JHEP 9811 (1998) 004, hep-th/9803050.

[21] J. A. Harvey and G. Moore, "Superpotentials and membrane instantons", hepth/9907026.

[22] P. M. Cowdall, "On gauged maximal supergravity in six dimensions", JHEP 9906 (1999) 018 [hep-th/9810041].

[23] H. Lu, C. N. Pope and P. K. Townsend, "Domain walls from anti-de Sitter spacetime", Phys.Lett. B391 (1997) 39, hep-th/9607164.

[24] H. Lu, C.N. Pope and J. Rahmfeld J, "A Construction for Killing Spinors on $S^{n}$ ", hep-th/9805151.

[25] Fagundes, "Closed Spaces in Cosmology", Gen. Rel. and Grav. 24 (1992) 199.

[26] S. Ferrara, J. A. Harvey, A. Strominger and C. Vafa, "Second-Quantized Mirror Symmetry", Phys.Lett. B361 (1995) 59, hep-th/9505162.

[27] S. Gubser, "Curvature Singularities: the Good, the Bad, and the Naked", hepth/0002160.

[28] A. H. Chamseddine and W. A. Sabra, "Magnetic strings in five dimensional gauged supergravity theories", Phys. Lett. B477 (2000) 329, hep-th/9911195.

[29] D. Klemm and W. A. Sabra, "Supersymmetry of black strings in D = 5 gauged supergravities", Phys. Rev. D62 (2000) 024003 hep-th/0001131. 